\title{
Building the Future Therapies for Down Syndrome: The Third International Conference of the T21 Research Society
}

\author{
Mara Dierssen $^{a, b, c}$ Yann Herault ${ }^{d}$ Pablo Helguera ${ }^{\mathrm{e}}$ Maria Martínez de Lagran ${ }^{\mathrm{a}, \mathrm{c}}$ \\ Anna Vazquez $^{b}$ Bradley Christian ${ }^{f}$ María Carmona-Iraguig, ${ }^{h} \quad$ Frances Wiseman $^{i}$ \\ William Mobley ${ }^{j}$ Elizabeth M.C. Fisher ${ }^{k}$ Veronique Brault ${ }^{d}$ Anna Esbensen ${ }^{\prime}$ Lisa M. Jacolam \\ Marie Claude Potier ${ }^{\mathrm{n}}$ Eric D. Hamlett ${ }^{\mathrm{O}}$ Leonard Abbeduto ${ }^{p}$ Laura del Hoyo Soriano ${ }^{p}$ \\ Jorge Busciglio ${ }^{q}$ Maria Florencia lulita ${ }^{r} \quad$ John Crispino $^{s}$ Sébastien Malinge ${ }^{t}$ \\ Eugenio Barone $^{\mathrm{u}} \quad$ Marzia Perluigi $^{\mathrm{u}} \quad$ Floriana Costanzo $^{\mathrm{v}}$ Jean Maurice Delabar ${ }^{\mathrm{n}}$

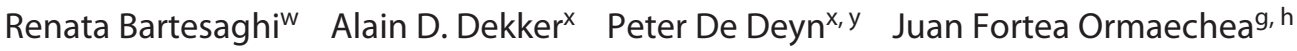 \\ Patricia A. Shaw ${ }^{z}$ Tarik F. Haydar ${ }^{z}$ Stephanie L. Sherman ${ }^{A}$ André Strydom ${ }^{B}$ \\ Anita Bhattacharyya ${ }^{f}$
}

${ }^{a}$ Centre for Genomic Regulation, Centre for Genomic Regulation (CRG), The Barcelona Institute of Science and Technology, Barcelona, Spain; bUniversitat Pompeu Fabra (UPF), Barcelona, Spain; 'Centro de Investigación Biomédica en Red de Enfermedades Raras (CIBERER), Barcelona, Spain; 'Université de Strasbourg, CNRS, INSERM, Institut de Génétique et de Biologie Moléculaire et Cellulaire, Strasbourg, France; eInstituto Ferreyra, INIMECCONICET-UNC, Córdoba, Argentina; 'Waisman Center, University of Wisconsin-Madison, Madison, WI, USA; ${ }^{9}$ Sant Pau Memory Unit, Department of Neurology, Hospital de la Santa Creu i Sant Pau, Biomedical Research Institute Sant Pau, Universitat Autònoma de Barcelona, Barcelona, Spain; ' ${ }^{B}$ Barcelona Down Medical Center, Fundació Catalana de Síndrome de Down, Barcelona, Spain; 'UK Dementia Research Institute, University College London, London, UK; iUniversity of California-San Diego, San Diego, CA, USA; kUniversity College London, London, UK; 'University of Cincinnati, Cincinnati Children's Hospital Medical Center, Cincinnati, OH, USA; mSt Jude Children's Research Hospital, Memphis, TN, USA; "Brain \& Spine Institute (ICM), CNRS UMR7225 - INSERM U1127 - UPMC Hôpital de la Pitié-Salpêtrière, Paris, France; ${ }^{\circ}$ Medical University of South Carolina, Columbia, SC, USA; PUniversity of California-Davis, Davis, CA, USA; 'University of California-Irvine, Irvine, CA USA; 'McGill University and Université de Montréal, Montréal, QC, Canada; ${ }^{5}$ Northwestern University, Evanston, IL, USA; ${ }^{T}$ Telethon Kids Institute - Cancer Centre, Nedlands, WA, Australia; "Sapienza University of Rome, Rome, Italy; 'Bambino Gesù Children's Hospital, Rome, Italy; 'Wniversity of Bologna, Bologna, Italy; ${ }^{\times}$University of Groningen and University Medical Center

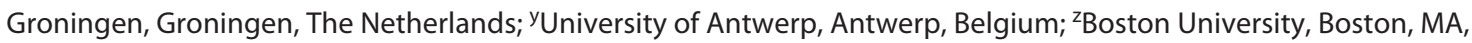
USA; ${ }^{A}$ Emory University, Atlanta, GA, USA; ${ }^{B}$ King's College London, London, UK

\section{Keywords}

Down syndrome $\cdot$ Intellectual disability · Language impairment · Mouse models · Neurodevelopmental disorders · Alzheimer's disease · Biomarkers · Trisomy 21 Research Society

karger@karger.com

(c) 2021 S. Karger AG, Basel

www.karger.com/msy

Karger"

\begin{abstract}
Research focused on Down syndrome has increased in the last several years to advance understanding of the consequences of trisomy 21 (T21) on molecular and cellular processes and, ultimately, on individuals with Down syn-
\end{abstract}

Correspondence to:

Anita Bhattacharyya, bhattacharyy@waisman.wisc.edu 
drome. The Trisomy 21 Research Society (T21RS) is the premier scientific organization for researchers and clinicians studying Down syndrome. The Third International Conference of T21RS, held June 6-9, 2019, in Barcelona, Spain, brought together 429 scientists, families, and industry representatives to share the latest discoveries on underlying cellular and molecular mechanisms of T21, define cognitive and behavioral challenges and better understand comorbidities associated with Down syndrome, including Alzheimer's disease and leukemia. Presentation of cuttingedge results in neuroscience, neurology, model systems, psychology, cancer, biomarkers and molecular and pharmacological therapeutic approaches demonstrate the compelling interest and continuing advancement in all aspects of understanding and ameliorating conditions associated with T21.

(c) 2021 S. Karger AG, Basel

Trisomy 21 (T21) causes Down syndrome (DS), the most common genetic cause of intellectual disability and the most recognized genetic syndrome. Individuals with DS and their families face significant health challenges. Scientific discoveries at the basic, preclinical and clinical levels are necessary to improve development and health of individuals with DS. In addition, research focused on DS can benefit typical individuals at risk for co-morbidities associated with DS.

The T21 Research Society (T21RS) was founded in 2014 to stimulate and facilitate preclinical and clinical DS research, support education and training for young researchers, and promote interaction between scientists, people with DS and their families. Biennial conferences are devoted to advancing both basic and clinical science in DS and provide a venue for presentation of exciting and innovative research on the varied impacts of DS. The Third International Conference of T21RS was held June 6-9, 2019 at Cosmo-Caixa in Barcelona, Spain. Building on the success of previous conferences in Paris and Chicago [Delabar et al., 2016; Reeves et al., 2019], the meeting engaged new researchers to the DS field and promoted collaboration with current researchers. 429 scientists, families, and industry representatives from around the world attended with 167 posters presented and 65 chosen for short talks. The meeting included 4 plenary lectures, 3 satellite symposia, 14 symposia, 1 Industry session, 1 Education Committee Session, 6 pre-meeting workshops, and a tour of the Barcelona Biomedical Research Park. A program for families, the Science \& Society Symposium and a Social Program complemented the research presentations.

Third International Conference of the T21 Research Society

\section{Human Chromosome 21 and Human Brain Development}

In the Lejeune Plenary Lecture, Stylianos Antonarakis (Switzerland) gave a historical perspective on human chromosome 21 (Hsa21) genes and their causative relationship to the characteristics of DS individuals. By comparing the transcriptional kinetics of a gene triplicated in T21, he showed that the presence of a doubled allele in trisomic cells leads to increased monoallelic expression compared to diploid cells and more simultaneous activation of gene expression compared to diploid controls [Stamoulis et al., 2019]. These data provide a deeper understanding of the causative links between altered gene expression and phenotypes in T21. Dr. Antonarakis was presented the Montserrat Trueta Outstanding Career Award to recognize his dedication to understanding the genetics of Hsa21 and DS.

Nereo Kalebic (Germany) gave the EMBO Plenary Lecture and discussed human-specific genes, neural stem cell amplification, and neocortical expansion in development and human evolution. He presented data showing neocortical expansion in humans compared to other species is due to increased proliferation of basal progenitors and identified human membrane-bound palmdelphin as a factor mediating progenitor expansion [Kalebic et al., 2019]. These results have implications for cortical development in DS, where progenitor neural proliferation and expansion is reduced.

\section{Models to Study DS}

The meeting included a "Mouse Master Class" workshop organized by the T21RS Preclinical Committee and presentations of findings using established mouse models and the introduction of new, innovative mouse models to the T21RS community that are likely to be widely used. Further, the establishment of human induced pluripotent stem cells (iPSCs) from somatic cells of DS individuals was also presented and examples of how this system enables elucidation of human-specific cellular and molecular characteristics underlying T21 were provided.

Investigating DS Phenotypes and Mechanisms through Comprehensive Mouse Modeling

The Ts65Dn model, due to its strong cognitive phenotypes and easy accessibility, has been the most widely used DS model for phenotypic analyses and testing of potential therapeutics. However, overexpression of additional trip- 
licated genes likely contribute to the phenotypes of Ts65Dn mice.

To address this problem, Yann Herault (France) presented the Ts66Yah mouse model which does not carry the trisomy of the centromeric Mmu17 region and recapitulates many behavioral phenotypes observed in the Ts65Dn model. Gene expression analysis of Ts66Yah compared to the Ts65Dn hippocampus revealed that the Ts66Yah hippocampus has several dysfunctional pathways compared to controls including some pathways similarly dysregulated in the Ts65Dn hippocampus, as well as distinct differences between the 2 strains.

Roger Reeves (USA) described a mouse model created with Drs. Yasuhiro Kazuki and Mistuo Oshimura using a mouse artificial chromosome containing most of the long arm of Hsa21 (MAC21). The MAC21 model is not mosaic and has similar gene expression, protein expression, and a number of phenotypes present in other mouse models of DS, validating the model for studies relevant to DS [Kazuki et al., 2020]. Interpretation of results from mouse models overexpressing human instead of mouse genes and the strengths and weaknesses of different model systems were discussed.

Randall Roper (USA) discussed modeling of skeletal abnormalities and comparison of several DS mouse models with differing trisomic content that revealed different trisomic genes and/or regions may exacerbate or improve bone mineral density, skeletal structure, stature and craniofacial skeletal phenotypes associated with DS and lead to the sexual dimorphism seen in DS. Understanding the gene-phenotype relationship in DS mouse models may be used to decrease fracture risk and design specific treatments to improve skeletal deficits in individuals with DS and other individuals with skeletal abnormalities.

Veronique Brault (France) showed correlation of DYRK1A overexpression with cognitive deficits. Dyrk1a plays a crucial role in the perturbation of the excitation/ inhibition (E/I) balance that leads to deficits in synaptic transmission and memory. Using a conditional knockout allele of Dyrk1a crossed with the Tg (Camk2-Cre) 4Gsc transgenic mouse that targets forebrain glutamatergic neurons, or with the $\mathrm{Tg}$ (dlx6a-cre) 1Mekk mouse that targets forebrain GABAergic interneurons Dyrk1a expressioncan be selectively knocked down in specific cell populations. Dyrk1a gene dosage change in glutamatergic neurons did not impact working memory or susceptibility to epilepsy, which was solely impacted by the Dyr$k 1 a$ gene dosage change in GABAergic neurons. Interestingly, Dyrkla gene haploinsufficiency in GABAergic neurons affected social behavior. However, long-term object recognition memory was impacted by Dyrk $1 a$ gene copy number in glutamatergic neurons, indicating a major effect of Dyrkla triplication in the excitatory neuron subpopulation in declarative memory. These findings point to a major impact of Dyrkla via a direct impact on GABAergic neurons and reveal that Dyrkla has a direct cell-type specific function in regulating long-term explicit memory in glutamatergic neurons.

Elizabeth Fisher (UK) showed altered hippocampalprefrontal neural dynamics in the Dp1Tyb mouse model through electrophysiology and the T-maze behavioral test. Dp1Tyb mice have a delayed decision-making phenotype that is associated with altered theta dynamics: reduced frequency, increased hippocampal-mPFC coherence, and increased modulation of hippocampal high gamma. Using Dp1Tyb*Dyrk1aKO mice that have only 2 functional copies of Dyrk1a, the group observed a similar delayed decision-making phenotype as seen in Dp1Tyb animals. Hence, a third copy of Dyrk1a is not necessary to produce this behavioral deficit in Dp1Tyb mice, and must therefore arise from other gene(s) in this region of Hsa21 homology. These results demonstrate that behavioral deficits associated with altered neuronal dynamics in a DS mouse model do not result from the increased expression of Dyrk1a.

\section{New Directions in Induced Pluripotent Stem Cells}

(iPSC) to Study DS

The power of human iPSCs to model human development lies in the ability to recapitulate many developmental steps to generate specific cell types in vitro over a period of time that corresponds to in vivo development. Engineering better stem cell models and exploring epigenomics of T21 are novel avenues of investigation of the consequences of T21 and may potentially identify new targets of therapeutics.

Stefan Pinter (USA) discussed efforts to understand the effect of T21 on neural differentiation and, in particular, the link between reported impaired primary cilia [Galati et al., 2018] and neural induction and rosette formation by neural progenitors. Dr. Pinter also presented new strategies to silence the extra Hsa21 using DOX-inducible XIST after neurogenesis, rather than at the pluripotent stem cell stage [Jiang et al., 2013].

Hiruy Meharena (USA) presented his work on the consequences of T21 on the epigenome in iPSC-derived neural cells. Even though numerous studies have recently shown genome-wide disruption of gene expression because of T21, the epigenomic consequences remain largely unexplored. Dr. Meharena discussed transchromo- 
somal interactions, specifically chromosome introversion, and presented data linking altered chromosomal looping with differentially expressed genes.

Ernst Wolvetang (Australia) used CRISPR genome editing to normalize APP gene copy number in DS iPSCs and showed that APP copy number has a surprisingly strong impact on genome wide and Hsa21-specific neuronal gene expression that is neurodevelopmental stage dependent. Neuronal differentiation into cortical neurons or brain organoids revealed APP gene copy number affects amyloid production and deposition but not Taupathology, promotes premature neuronal differentiation, and enhances glial differentiation at the expense of neurogenesis. Single cell RNA seq corroborated these findings and identified cell type-specific overexpression of different cohorts of HSA21 genes at specific developmental stages [Ovchinnikov et al., 2018].

Melvys Valledor (USA) presented a short talk on her work using CRISPR genome editing to establish isogenic T21 iPS cell lines that vary in the gene dosage of APP, including a null line. She used these valuable lines to generate cerebral organoids and presented her RNA and protein expression data that nicely complemented the results from Wolvetang, providing critical reproducibility in the human stem cell field.

Blandine Ponroy (Canada) explored the molecular and cellular differences in astrocytes differentiated from T21 iPSCs and showed that T21 astrocytes have transcriptional and chromatin accessibility differences compared to control cells. Misexpression of neurodevelopmental, cell adhesion, and extracellular matrix genes were concomitant with perturbations in astrocyte size, cell-cell and cell-substrate adhesion, and protocadherin (PCDH)mediated cell recognition. Thus, T21 leads to global epigenomic and transcriptomic changes in astrocytes that affect astrocyte adhesive properties [Ponroy Bally et al., 2020].

\section{Advantages and Challenges of Mouse Models and Human iPSCs to Study DS}

Researchers using different models came together during the Education Committee Workshop to discuss the existing model options to study DS and present ongoing in vivo and in vitro approaches driving future discovery.

Mouse models have been of vital importance to study and understand many aspects of DS. Nevertheless, significant differences exist at the molecular and systems levels between rodents and primates. Elizabeth Fisher (UK) pointed out that the scientific objective should be the main driver of which model is best suited for the re- search at hand, opting for an integrative methodology that incorporates both in vivo as well as in vitro models [Fisher and Bannerman, 2019].

Victor Tybulewicz (UK) suggested using multiple models to verify findings and identify consistent phenotypes in the mice. Some models take advantage of the portions of mouse chromosomes 10, 16, and 17 syntenic to Hsa21 while others contain a copy of Hsa21 itself. While models containing human genes from $\mathrm{Hsa} 21$ can incorporate the full range of genetic material triplicated in DS, introducing human-specific genes into the mouse may not accurately reproduce the effect of those genes in the human environment. Conversely, when utilizing the triplication of mouse genes, the mouse orthologues may have different levels of expression and yield different phenotypes than those found in people with DS.

Randall Roper (USA) discussed the impact of method of generation and/or genetic background on phenotypes. For example, when Ts1Rhr is bred on the inbred C57BL/6 background, trisomic animals have an increased volume of neural crest first pharyngeal arch (PA1) region during embryonic development and a reduced body weight during adulthood. However, on a mixed C57BL/6-C3H background the model shows no difference in the volume of PA1 and the trisomic animals have an increased adult body weight compared to controls. These examples show that mouse model husbandry is a critical variable to consider.

Tarik Haydar (USA) chronicled the changing phenotypes of Ts65Dn over generations. His lab's findings illustrate that even animals of the same trisomic line bred on the same background but of different historic times and generation exhibit significant variation in well-documented phenotypes, including cortical expansion during embryonic development, the trajectory of oligodendrogenesis and maturation, and learning and memory performance. The phenotypes also showed a drift in wild type mice. Dr. Haydar's work suggests there may be a greater degree of variability than previously anticipated and that the phenotypes of trisomic animals may not be preserved from generation to generation [Shaw et al., 2020] and raises the idea that variability between animals may be a representation of the variability within the DS population.

Human models that recapitulate human-specific genetics and brain development are alternatives to using mouse models to study DS. Jeanne Lawrence (USA) and Anita Bhattacharyya (USA) emphasized the value of iPSCs generated from individuals with DS and the use of brain organoids to study neural development. While iP- 
SCs accurately represent the human genetic environment, in vitro experiments do not recapitulate the environment of a developing brain. Cell lines differ from lab to lab, and differences in culturing techniques can affect how the cells develop in vitro. Drs. Lawrence and Bhattacharyya called for a greater degree of transparency between labs using iPSCs, including the sharing of isogenic cell lines and detailed protocols to mitigate variances in experimental outcomes.

\section{Cellular and Mechanistic Insight into DS}

\section{New Disease-Relevant Behavioral Perspective in \\ Preclinical Intellectual Disability Studies}

In this symposium, Nicholas Santiago (USA) presented a new approach to study hippocampus-dependent learning and memory to avoid interpretive cofounding factors. Using the social transmission of food preference (STFP) paradigm, Ts65Dn mice showed normal learning and memory, but did not retain socially acquired information, evidenced by impaired performance following the 7-day retention interval. The STFP paradigm can be a valuable tool for studies using DS models to evaluate potential therapies targeting hippocampal function.

Charles Hoeffer (USA) explored the sleep abnormalities common in DS individuals using the Dp16 mice that show altered electroencephalography (EEG) oscillations in both sleep and awake states. Aged mice showed disrupted sleep characterized by increased activity during the dark phase and increased time awake at the expense of Non-Rapid Eye Movement (NREM) sleep, similar to DS individuals. Correcting the dosage of Regulator of Calcineurin1 (RCAN1), a Hsa21 gene, ameliorated some sleep and EEG abnormalities, suggesting that underlying differences in neuronal activity at the network level might be causative rather than merely symptoms of DS.

Victor Tybulewicz (UK) presented the Dp1Tyb mouse model with a segmental duplication of the Hsa21-orthologous region of mouse chromosome 16 (MMU16), and a series of 8 other strains with shorter duplications that can be used to identify the location of causative dosage-sensitive genes. Dp1Tyb mice showed embryonic heart malformations, disrupted sleep, memory problems, craniofacial alterations, a pre-diabetic state and splenomegaly, similar to phenotypes seen in DS. Interestingly, the locomotor defects of Dp1Tyb mice mapped to a 25-gene interval and one of the causative genes was Dyrkla. His results showed the Dp1Tyb mice are a good model to explore DS phenotypes.
Eugene $\mathrm{Yu}$ (USA) described functional interactions among different genetic elements of the Setd4-Mx2 region syntenic to Hsa21 based on the cognitive profile of four new mouse mutants. Dr. Yu initiated a genetic dissection of the mechanisms of DS-specific differential methylation, providing two candidate mechanisms, one involving altered expression of genes in S-adenosylmethionine-dependent methylation pathways and the other involving altered occupancy of transcription factor binding sites in cells with T21.

Mahiuddin Ahmed (USA) presented a new potential therapy to revert cognitive deficits in DS and physiological aging. Administration of Granulocyte-macrophage colony-stimulating factor (GM-CSF) in Dp(16)1Yey/+ (Dp16) mice reversed some behavioral deficits in the radial arm maze and brain neuropathology, specifically in astrocyte morphology and inhibitory neuron populations.

\section{Stereotyped Homeostatic Compromises}

Despite their strikingly different genetics, human autosomal trisomies 13,18 and 21 share dysfunctional phenotypes that may shed light on potential therapeutic targets for DS. In particular, accelerated senescence in human trisomic lines has been associated with different aspects of altered cellular metabolism.

Eduardo Torres (USA) focused on nuclear morphological alterations caused by long chain lipids deficit shared by all human autosomal trisomies. Lipid-related nuclear disruption was recapitulated in multiple lines of artificially generated yeast with single supernumerary chromosomes. Interestingly, increased levels of long chain fatty acids rescued nuclear morphology and cellular fitness both in human and yeast aneuploid cells.

Jorge Busciglio (USA) described a pattern of mitochondrial dysfunction and oxidative stress characterized in T2 1 cells and extended to trisomies of chromosome 13 and 18 respectively. Oxidative damage correlated with increased mitophagy, while concomitantly triggering a common Nrf2-mediated antioxidant response. The induction of an enhanced Nrf2 response by treatment with Oltipraz prevented accelerated aging phenotypes in all 3 human trisomies, suggesting that the modulation of the Nrf2 pathway represents a potential target for pharmacological intervention [Hwang et al., 2019].

Yasuji Kitabatake (Japan) demonstrated detrimental effects in trisomic cells predicted by general aneuploidyassociated stress. In particular, he focused in abnormal accumulation of protein aggregates and increased apoptosis in fibroblasts and iPSCs. Treatment with sodium 
4-phenylbutyrate (4-PBA), a chemical chaperone, prevented protein aggregate deposits and associated pathological phenotypes [Nawa et al., 2019].

The session included 2 brief talks by Mohit Rastagi (Italy) who presented advances in the identification of targets for cognitive impairment in DS applying integrated multi-omics-driven system biology and Chiara Di Vona (Spain) that described a novel link between Dyrk1a to RNF169 and DNA damage response using proteomics based interaction screen.

\section{Modifications of Autophagy-lysosomal-endosomal \\ Pathways in DS and other Neurodegenerative \\ Disorders.}

The morphology and functional properties of several organelles are altered in DS and other neurodegenerative diseases. The endo-lysosomal pathway is modified in Alzheimer disease $(\mathrm{AD})$ and $\mathrm{DS}$ while lysosomal-autophagy pathway dysfunctions are common mechanism in neurodegeneration.

Steve Finkbeiner (USA) presented an automated imaging platform capable of high-throughput longitudinal single-cell analysis to characterize the autophagy-lysosomal pathway (ALP). Dr. Finkbeiner's lab imaged the clearance of misfolded proteins in single neurons and showed that deficits in protein homeostasis could predict neurodegeneration [Finkbeiner, 2020]. These deficits could be corrected by specific compounds selected using this automated technology. Deficits in the ALP may represent therapeutic targets that are applicable to multiple neurodegenerative diseases including DS-related AD.

Andre Miranda (Portugal) presented studies on phosphatidylinositol-3-phosphate (PI3P) that is synthesized primarily by class III PI 3-kinase called Vps34. Disruption of Vps34 function leads to early endosomal enlargement and blockade of autophagy via inhibition of autophagosome formation as well as an accumulation of amyloid precursor protein C-terminal fragments (APPCTFs) and a counteracting homeostatic response involving elevated secretion of atypical extracellular vesicles (exosomes) [Miranda et al., 2018b]. In response to a VSP34 defect, these exosomes help eliminate lysosomal waste, including pAPP-CTFs and glycerophospholipids, like bis(monoacylglycero)phosphate (BMP). These exosomal products may be useful candidate biomarkers for endolysosomal dysfunction associated with neurodegeneration in DS.

Catherine Marquer (USA) explored the role of the regulator of synaptic vesicle trafficking SYNJ1, a Hsa21 encoded protein linked to amyloid toxicity and endolyso-

Third International Conference of the T21 Research Society somal dysfunction in AD. Increased SYNJ1 levels in autopsy brains from adults with DS/AD were inversely correlated with synaptophysin levels, a read-out of synaptic integrity. Mice overexpressing Synj1 exhibited hippocampal hyperexcitability and defects in the spatial reproducibility of place fields, thus highlighting a novel role for SYNJ1 in the function of place cells in memory deficits [Miranda et al., 2018a]. Results suggest elevated levels of SYNJ1 in DS-related AD and sporadic AD brain tissue could reflect synaptic deficits and long-term memory decline.

Beverly Rothermel (USA) discussed the role of another Hsa21 encoded gene, calcineurin1 (RCAN1/DSCR1), on mitochondrial function. Using human iPSCs, she showed that T21 iPSCs had increased mitochondrial fusion and elevated metabolic activity compared to isogenic controls. Depletion of RCAN1 from T21 iPSCs was sufficient to restore the mitochondrial network, reducing both $\mathrm{O}_{2}$ consumption and ROS generation. The hyperfused mitochondrial network observed in T21 suggests that mitochondrial dysfunction may be due to increased RCAN1 suppression of mitochondrial fission [Parra et al., 2018].

\section{Correction of Circuit-Specific GABAergic}

Overinhibition and Dendritic Alterations in DS

Increasing studies converge on the hypothesis that excitatory-inhibitory imbalance occurs in DS and affects synaptic plasticity and cognitive performance.

Trevor G. Smart (UK) described an increased frequency of GABA-mediated spontaneous inhibitory postsynaptic currents (sIPSCs) and increased levels of tonic inhibition in dentate granule cells in acute brain slices of the Dp1Tyb mouse model compared to controls, without changes to sIPSC amplitudes. These results are consistent with increased GABA-mediated inhibition in trisomic neurons likely to be driven by presynaptic mechanisms. Resting membrane potentials between trisomic and disomic neurons were similar, but the threshold for spike firing was increased for trisomic cells. Using fluorophore filled single hippocampal neurons, trisomic neurons undergo dendritic remodeling that may involve changes to parvalbumin interneurons that may underlie the increased phasic and tonic inhibition.

Javier Zorrilla de San Martin (France) reported in vivo single unit and local field potential recordings from the prefrontal cortex of Ts65Dn mice and whole-cell patch clamp recordings from specific neuronal types composing cortical inhibitory circuits. While in vivo recordings revealed a high degree of network desynchronization in 
Ts65Dn mice, simultaneous whole-cell patch clamp recordings from layer $2 / 3$ pyramidal neurons (PNs) and dendrite targeting Martinotti cells (MCs) revealed a boosted activity of this inhibitory feedback loop due to enhanced synaptic strength in trisomic mice. Perisomatic inhibitory connections made by parvalbumin interneurons are not altered in trisomic mice while their excitability and characteristic fast spiking activity is dramatically altered leading to an overexcitable PV phenotype. These results show the existence of 2 circuit-specific mechanisms.

Jan Michael Schulz (Switzerland) described local field potential and whole-cell patch-clamp recordings from the CA1 region of hippocampal brain slices from Ts65Dn mice. IPSCs onto dendrites of pyramidal neurons evoked at increasing stimulation intensities in stratum radiatum (SR) were significantly larger in Ts65Dn. By contrast, there was no difference in perisomatic IPSCs evoked in stratum pyramidale, but a $\sim 25 \%$ reduction of NMDARmediated PSCs relative to AMPAR-PSCs in SR. Consequently, burst-induced NMDAR-mediated membrane depolarization and long term potentiation (LTP) induction are impaired in Ts65Dn mice. a5-GABAARsmediated IPSCs evoked in SR showed a nonlinear voltage dependence and slow decay time constants closely matching NMDAR-PSCs in wild-type and Ts65Dn mice. Selective pharmacological reduction of a5-GABAAR-mediated inhibition rescued both, synaptic NMDAR activation and LTP, suggesting both underlie impaired synaptic plasticity in Ts65Dn mice.

Vincenzo de Paola (UK) showed human iPSC-derived cortical neurons transplanted in the adult mouse cortex organized in large vascularized neuron-glia territories with complex cytoarchitecture. Longitudinal imaging of more than 4,000 grafted neurons revealed that neuronal arbors refined via branch-specific retraction; human synaptic networks substantially restructured over 4 months, with balanced rates of synapse formation and elimination, and oscillatory population activity mirrored the patterns of fetal neural networks. Using T21 iPSC-derived neurons, dendritic spines were more stable and tended to be more abundant in T21 circuits compared to controls, and there was a reduction in nascent oscillatory network activity in T21 [Real et al., 2018].

Anita Bhattacharyya (USA) described the analysis of interneuron subtypes in postmortem superior temporal gyrus from adult DS individuals. No difference was observed in the parvalbumin population between DS and controls, but the density of calretinin cells was reduced in DS. Using isogenic T21 iPSCs patterned to interneuron progenitors, she confirmed that fewer COUP-TFII + progenitors, that give rise to calretinin neurons, are generated from T21 iPSCs. Further, microarray and single-cell analyses indicate that $\mathrm{T} 21$ progenitors have altered response to $\mathrm{SHH}$ and WNT signaling pathways, providing mechanistic insight into the generation of fewer COUPTFII progenitors and calretinin neurons in DS.

\section{Metabolic Defects in DS: From Periphery to the Brain}

Growing evidence supports the role of metabolic defects as a risk factor for cognitive impairment in DS. Defining the molecular mechanisms underlying these processes in DS is a key challenge to identify novel drug targets and establish prevention strategies to reduce the impact of metabolic disorders on cognitive decline [Dierssen et al., 2020].

Aurelie Ledreux (USA) discussed reliable blood biomarkers to improve early diagnosis and as readouts for treatment trials and proposed extracellular vesicles (EVs) secreted into the blood and circulate to cellular targets. Dr. Ledreux focused on the isolation of neuronal EVs (NDE) to quantify AD biomarkers (Tau, phospho-Tau, amyloid beta (A $\beta$ ) 40, A $\beta$ 42, BDNF, neurofilament-light) in individuals with $\mathrm{DS}$ and compared $\mathrm{AD}$ biomarker levels between cerebrospinal fluid (CSF) and neuron-derived EVs from the same individuals to validate neuronderived EV biomarker levels as diagnostic tools for DSAD [Hamlett et al., 2017].

Kelly Sullivan (USA) presented the results of a large plasma metabolomics study, which revealed the kynurenine $(\mathrm{KYN})$ pathway $(\mathrm{KP})$ of tryptophan catabolism is activated in DS subjects, resulting in higher levels of KYN and the neurotoxic metabolite and quinolinic acid QA. Expression of IDO1, the interferon-inducible enzyme catalyzing the rate-limiting step of this pathway is upregulated in circulating immune cells of people with DS. Using cell culture models coupled with metabolic tracing assays Dr. Sullivan showed that DS cells are hypersensitive to both induction of IDO1 levels and activation of the $\mathrm{KP}$ and that increased interferon receptor copy number is required for this hypersensitivity [Powers et al., 2019].

Maria Chiara Pelleri (Italy) reported results of nuclear magnetic resonance (NMR) analysis profiling the metabolome in plasma and urine samples from DS subjects. The multivariate analysis o showed a clear discrimination (up to of $80 \%$ accuracy) between the DS and control groups. Most of the altered concentrations were consistent with the 3:2 gene dosage model, suggesting effects caused by the trisomy 21: DS/normal ratio in plasma was 1.23 (pyruvate), 1.47 (succinate), 1.39 (fumarate), 1.33 (lactate), 
1.4 (formate). Several altered metabolites are produced in the Krebs cycle. More analyses are necessary to identify the mechanism that underlies the unbalanced concentration of metabolites [Antonaros et al., 2020].

Eugenio Barone (Italy) showed that DS brain recapitulates key features of brain insulin resistance similar to the AD brain. Evaluation of neuronal-derived exosomes (NDE) showed that DS subjects have reduced insulin receptor (IR) levels and increased inhibitory phosphorylation of insulin receptor substrate-1 (IRS1), compared to age-matched controls. Similar changes for IR and IRS1 were observed in cortical postmortem brain samples from DS and controls. Alterations of brain insulin signaling parallel loss of synaptic proteins suggesting reduced downstream neuronal plasticity mechanisms in DS and suggest that alterations of brain insulin signaling may contribute to dementia in DS subjects [Tramutola et al., 2020].

\section{Cognition and Language in Individuals with DS}

\section{Cognitive Outcomes in Children with DS}

Individuals with DS often present with a distinct pattern of behavioral and cognitive skills, but there is variability across individuals in different domains, including self-regulation, social-emotional functioning, cognition, attention, and language and motor skills. There is a need to establish empirically evaluated, psychometrically strong outcome measures appropriate for use in this population.

Lisa Jacola (USA) presented findings from a study of neurocognitive outcomes in survivors of childhood acute leukemia with DS. A comprehensive test battery included research measures of attention, executive functioning, and processing speed and standardized parent ratings of attention and executive function. Results supported the clinical utility of the research battery. For example, worse performance on measures of verbal fluency significantly predicted elevated parent-rated symptoms of attention problems and executive dysfunction. Compared to previously collected data from an age-similar cohort of individuals with DS without cancer, the DS-leukemia cohort had lower semantic fluency scores. Overall, these results support the feasibility and clinical utility of a novel approach to neurocognitive assessment in individuals with DS-leukemia.

Silvia Lanfranchi (Italy) discussed the heterogeneity in cognitive profiles of children with DS, not only to assess strengths and weaknesses, but also to understand the sources of individual differences. Administering the WPPSI III scales to 60 children with DS, she found no significant differences between verbal and nonverbal indices, yet high variability within participants. Further, cognitive indices correlated with age, language development, and adaptive functioning. Cluster analysis revealed 3 different subgroups: (1) the classical profile of higher nonverbal than verbal abilities, (2) the opposite profile of higher verbal than nonverbal abilities, and (3) overall high performance on both verbal and nonverbal abilities with no differences between abilities. These data support the hypothesis that, together with genetic factors, other interacting factors are likely to contribute to individual differences and cognitive-level outcomes in DS.

Anna Esbensen (USA) presented findings on the concurrent relationship between parent-reports of executive functioning and parent-reports of maladaptive behavior among children with DS, and replicated the concurrent relationship with teacher-reports. Parent- and teacherreported concerns for behavioral regulation were related to parent- or teacher-reported concerns for inattention, rule-breaking behavior, and aggressive behavior. In contrast, parent- and teacher-reported concerns for metacognition were only related to concerns of inattention. The results suggest a consistent pattern of relationships between executive functioning and maladaptive behaviors across settings. Understanding the relationship between these constructs has important implications for appreciating the contributing factors to presenting maladaptive behaviors, early targets of intervention, and developing preventative strategies.

\section{Language Outcome Measures for Pharmacological} and Behavioral Intervention Studies

Difficulties in language and communication are ubiquitous among individuals with DS and declines in these domains co-occur with the onset of AD symptoms. Consequently, pharmacological and behavioral interventions targeting language and communication are needed; however, evaluation of these interventions is being hampered by a lack of adequate outcome measures [Esbensen et al., 2017].

Angela John Thurman (USA) described an ongoing multi-university consortium designed to evaluate a variety of prelinguistic communication and spoken language metrics for use in treatment studies focused on young children with DS ages 2-8 years of age. The project is using natural communication sampling, which entails recording and analyzing the frequency and quality of communication of young children with DS in a variety of structured and unstructured play activities. 
Leonard Abbeduto (USA) described an ongoing multiuniversity consortium that is documenting the utility of expressive language sampling (ELS) as a source of psychometrically sound outcome measures for treatment studies. ELS entails collecting samples of spoken language from participants interacting with an examiner in naturalistic, yet standardized, interactions involving individuals with DS, ages 6-23 years.

Rafael de la Torre Fornell (Spain) presented data in support of the semantic verbal fluency task (SVFT) as a useful tool for screening for the early signs of dementia and amyloidosis in young adults with DS. In this task, the participant generates as many words as possible from a specific category (e.g., animals).

Natalia Arias Trejo (Mexico) presented results of a study documenting the ability of people with DS to use the information provided by a verb to anticipate, or predict, the referential properties of a subsequent word in a sentence during oral language comprehension. This skill was also found to be an indicator of more general changes in cognitive functioning and thus, has potential as an indicator of treatment response.

Margaret Pulsifer (USA) described a study conducted at three US sites that found that language skills, especially in the receptive domain, can predict AD-related cognitive decline in adults with DS. Collectively, these projects offer a new set of tools for measuring improvements in treatment studies and marking points of AD-related decline at which intervention is needed.

\section{Health Comorbidities in Individuals with DS}

\section{Health Comorbidities in Children with DS}

A pre-meeting session was organized by the T21RS Child Developmental Clinical Committee to introduce current clinical gaps in knowledge that can be addressed through clinical research participation. Stephanie Sherman (USA) introduced the importance of clinical research participation and discussed new resources and technological advances to better understand the biological underpinnings of the variation in clinical outcomes in DS, with a goal of reducing the effects of T21. She provided a genotype/phenotype study as an example to identify factors that modify the severity of congenital heart defects.

George Capone (USA) discussed medical conditions that negatively affect cognition and behavior during childhood, including infantile spasms, severe sleep apnea, and visual-hearing impairments. Dr. Capone emphasized that some of the most severe neurobehavioral conditions (e.g., regression, autism, stereotypy/self-injury) typically observed in older children, adolescents, or young adults, often occur in the apparent absence of a well-defined medical condition. He proposed that the brain's intrinsic vulnerability due to T21 increases the susceptibility to neurobehavioral and psychiatric symptoms and extends throughout the neuro-maturational timetable, starting prenatally and extending through adolescence. It is likely that specific brain circuits are underpowered, underperforming, and trophically malnourished, leading to a heightened risk for functional decompensation due to either medical conditions or the failure of neurobiological events.

Floriana Costanzo (Italy) discussed psychiatric comorbidities of childhood/adolescents and their association with cognitive impairment, including Applied Behavior Analysis, cognitive-behavioral treatments, standard behavioral approach, and counseling and/or pharmacological treatment. A specific psychological, educative and/or pharmacological treatment is necessary, one that considers cognitive, adaptive and psychopathological facets of DS and takes into account specific strengths and weaknesses. Identification of genetic, neurological, and psychosocial factors that place some young people with DS at high risk for severe psychiatric disorders is critical.

Rafael de la Torre Fornell (Spain) discussed possible interventions from early childhood to adolescents for DS. Brief, early pharmacological interventions that may modify disease progression. It is important to consider: (i) the duration of interventions (chronic treatments vs. early short interventions), (ii) therapeutic effects seen in children may be lost in adults, (iii) prenatal interventions in DS may have some potential. Treatments enhancing the altered synaptic plasticity postulated in DS may be useful and include pharmacological (e.g., GABA, endocannabinoids, DYRK1A) and non-pharmacological (e.g., noninvasive brain stimulation, such as transcranial directcurrent stimulation) options. Therapeutic developments should be combined with functional cognitive training to maximize their effectiveness.

\section{Health Comorbidities in Older Adults with DS -}

\section{Assessment and Diagnosis}

A pre-conference symposium focused on contemporary health issues in adults with DS was organized by the T21RS clinical committee and chaired by André Strydom (UK).

Anne-Sophie Rebillat (France) presented clinical data on obstructive sleep apnea (OSA), a modifiable risk factor 
of cognitive dysfunction. Dr. Rebillat reported that $77.4 \%$ of 31 adults with DS had OSA, and $48.4 \%$ had severe OSA. Results were similar to previously reported systematic screening for OSA in 47 non-symptomatic individuals, of whom $72.3 \%$ had OSA including $31.9 \%$ with severe OSA in Europe [Giménez et al., 2018] and in the USA [Cornacchia et al., 2019]. These data confirm that subjective evaluation underestimates the presence of apneas in individuals with DS and that objective sleep measures and systematic screening are essential. Therapies and management include lifestyle changes well as continuous positive airway pressure (CPAP). CPAP increases quality of life of individuals with OSA, with or without DS, but there are significant compliance issues and further research is required to adapt CPAP to the needs of DS individuals and their families.

Tonnie Coppus (the Netherlands) presented data from their longitudinal study of aging adults with DS. DS individuals start to age prematurely compared to controls and can be considered "old" at the age of $40-45$, due to the development of typical aging-related comorbidities. However, clear data on the prevalence of chronic diseases and conditions (and their treatments) are lacking, and further epidemiological studies are needed to identify risk factors. Nevertheless, recent research confirmed that activities of daily living are impaired by these comorbidities, and there is a need for evidence-based and standardized care to better support better management of multimorbidity to improve the quality of life and survival of people with DS [Coppus, 2017].

Sharon Krinsky-McHale (USA) discussed findings from their longitudinal study on the cognitive profiles of older individuals with DS and how to distinguish the changes of dementia-free individuals from those exhibiting changes due to dementia [Krinsky-McHale and Silverman, 2013]. Results show that a wide array of neuropsychological measures that demonstrate utility in distinguishing individuals by dementia status and emphasized that it is necessary to factor in lifelong cognitive functioning when dementia status is being evaluated [KrinskyMcHale et al., 2017]. They concluded that working definitions of mild cognitive impairment-DS and AD-DS are needed referenced to individual decline rather than population-normed performance.

Mary McCarron (Ireland) reviewed dementia management and maintenance of functional abilities of older individuals with DS and highlighted the need for a support model that included counseling; psychological and medical surveillance with regular adjustments to care plans, end-of-life care plans, and supports to caregivers [Dodd et al., 2018]. Health and care organizations should

Third International Conference of the T21 Research Society recognize the greater likelihood of physical symptoms, comorbidities, immobility, and neuropathological deterioration to ensure ongoing high-quality care while community-based innovations could improve the quality of life [McCarron et al., 2018].

\section{Leukemia in Children with DS}

Children with DS have an increased risk of developing leukemia, higher treatment-related morbidities, and increased rate of relapses compared to typical children. Sébastien Malinge (Australia) discussed the relationship between DS and B-cell leukemia. Children with DS have a 27-fold increased risk of developing B-cell acute lymphoblastic leukemia (B-ALL). Dr. Malinge characterized 29 primary B-ALL T21 samples, including 8 DS-ALL, and found a high incidence of somatic mutations leading to RAS/MAPK pathway activation in 75\% (22/29) of B-ALL samples with T21. In vitro, overexpression of the constitutively active mutant $\mathrm{KRAS}^{\mathrm{G} 12 \mathrm{D}}$ was found to cooperate with T21 to increase the clonogenic potential of murine CFU-preB colonies, and alter transcriptional programs of hematopoietic stem cell and B cell differentiation. In preclinical studies, inhibition of the kinases MEK1/2, downstream of RAS, decreases leukemia burden in DS-ALL PDX models, and synergizes with conventional chemotherapeutic agents such as Vincristine, to further enhance their survival [Laurent et al., 2020]. The establishment of a comprehensive cohort of DS-ALL models showed that adding the MEK1/2 inhibitor trametinib to current chemotherapy is a promising strategy to improve the outcome of children with DS who have B-cell leukemia.

Jeanne Lawrence (USA) addressed the value of XISTbased "trisomy silencing" as an experimental approach to understand DS pathogenesis and to the longer-term prospect that "chromosome therapy" could be biological feasibility. In hematopoietic differentiation of T21 iPSCs, induced XIST expression and trisomy silencing consistently corrected the overproduction of megakaryocytes and erythrocytes, which underlies high risks of transient myeloproliferative disorder and leukemia in DS. Additionally, XIST-based trisomy silencing in neurons, rather than at an early stem cell stage, resulted in chromosome silencing. Hsa21 mRNA levels were fully reduced to disomic levels in both neural stem cells and neurons, demonstrating that pluripotency is not required to substantially dosage compensate T21 [Czerminski and Lawrence, 2020].

Andrew Lane (USA) presented recent work using DS mouse models to study HMGN1, linked to acute leukemia. In addition to the markedly increased risk of leuke- 
mia in constitutional T21, he discussed that among children and adults with ALL and AML but without DS, somatic gain of $21 \mathrm{q} 22$ is a recurrent acquired DNA copy number variant. HMGN1 is a chromatin remodeling protein that binds to nucleosomes to modulates chromatin accessibility and gene expression. In loss-of-function experiments in Ts1Rhr and Ts65Dn mice, HMGN1 was critical for self-renewal of lymphoid progenitors and promotion of leukemia. HMGN1 promotes amplification of lineage-specific transcriptional programs at the chromatin by increasing accessibility. Using multiplexed, indexed T7 ChIP-seq (Mint-ChIP), H3K27 acetylation at promoters and enhancers was particularly amplified by HMGN1 overexpression. Analysis of HMGN1 activity in hematopoietic stem and progenitor cells showed that HMGN1 promoted clonal advantage in hematopoietic stem cells and impaired myeloid differentiation. Dr. Lane showed new therapeutic targeting of HMGN1 effects on histone modifications via inhibition of the histone acetyltransferases $\mathrm{CBP} / \mathrm{p} 300$, which reversed the abnormal myeloid differentiation in T21 models.

\section{DS and Alzheimer's Disease (AD)}

Within the past 25 years, the average lifespan for a person with DS has doubled, from 30 to 60 years and with the increased lifespan has come the realization that people who have DS are at significantly elevated risk of developing early onset AD [Wiseman et al., 2015].

Li-Huei Tsai (USA) gave a plenary lecture focused on gamma oscillations $(30-90 \mathrm{~Hz})$ that are associated with higher-order cognitive functions and are disrupted in several AD mouse models. Dr. Tsai has shown that gamma oscillations can be evoked noninvasively with optogenetics via a light programmed to flicker at $40 \mathrm{~Hz}$ [Iaccarino et al., 2016]. Gamma oscillations could be restored to normal levels in $\mathrm{AD}$ mouse models using this method and changes in $\mathrm{AD}$-associated pathology, including cellular changes and reduced phospho-Tau and decreased amyloid plaques accompanied the normalization of gamma oscillations. Further, mice had improved spatial and recognition memory in response to increased gamma oscillations [Martorell et al., 2019]. These data present a compelling model for therapeutic intervention to improve cognitive function.

\section{Neuroimaging Biomarkers for $A D$ in $D S$}

Despite early evidence of $A D$ pathology, a wide variation in age at onset of dementia exists. Neuroimaging techniques provide the opportunity to detect these pathologies and track their trajectory over the course of disease development.

Sigan Hartley (USA) discussed the association between cognitive functioning and positron emission tomography (PET) $\beta$-amyloid in adults with DS prior to the onset of clinical AD. As part of the Alzheimer's Biomarkers Consortium - DS (ABC-DS) study, DS participants were administered an extensive neuropsychological battery and neuroimaging scans of brain structure, function and AD-related pathology. Decline in cognitive functioning started in the $40 \mathrm{~s}$, becoming more marked in the 50 $s$ for episodic memory, working memory language (both expressive and receptive), adaptive living, motor coordination and planning, and visuospatial construction. Longitudinally, PET $\beta$-amyloid accumulation was related to an accelerated rate of decline across time points for expressive and receptive language, episodic memory, visual attention, visuospatial construction, and motor planning and coordination. Establishing this connection between preclinical $\mathrm{AD}$ biomarkers and declines in cognitive functioning will provide critical information for guiding therapeutic trials for the prevention of $\mathrm{AD}$ in the $\mathrm{DS}$ and general populations.

Adam Brickman (USA) discussed the distribution of magnetic resonance imaging (MRI)-derived cerebrovascular lesions in older individuals with DS and comparison across diagnostic categories (unaffected, MCI-DS, dementia) in the ABC-DS study. Because individuals with DS have relatively low prevalence of vascular risk factors, such as hypertension and diabetes, this provides an opportunity to study cerebrovascular disease in $\mathrm{AD}$ without confounds of systemic vascular disease. DS individuals have dramatic differences in cerebrovascular markers across the diagnostic groups. Elevated $\mathrm{WMH}$ volume, a greater prevalence of infarcts and cerebral microbleeds, and enlarged perivascular spaces associated with MCI-DS and dementia are present in DS compared to the unaffected group. Thus, cerebrovascular disease is a driver of $\mathrm{AD}$-related symptoms, and perhaps pathogenesis, and point to new possible therapeutic targets.

Elizabeth Head (USA) introduced studies of the presence of microbleeds in the DS brain and their relationship with cerebral amyloid angiopathy (CAA) and $\beta$-amyloid. Brain autopsies revealed CAA was more extensive in cases of DS dementia in the frontal and occipital cortex than that observed in sporadic AD (non-DS) and age-matched controls. Microbleeds are also more prevalent in DS dementia compared to controls but similar to sporadic AD and are seen after age 30 years in DS, and their presence 
rapidly increases in a regionally dependent matter. Cerebrovascular neuropathology may be a critical factor driving dementia in people with DS and may interact with $\mathrm{AD}$ neuropathology to lead to earlier ages of onset or more rapid progression.

Michael Rafii (USA) presented results from the DS Biomarker Initiative that demonstrated that plasma NF-L levels correlate with standard biomarkers of AD pathology such as amyloid PET, Tau PET, hippocampal atrophy as well as cognitive and functional decline. Plasma NF-L correlated in a statistically significant way with amyloid load (as assessed with florbetapir PET) as well as with markers of neurodegeneration (regional cerebral glucose metabolism as assessed with fluorodeoxyglucose (FDG) PET and hippocampal atrophy). Specifically, in regions that are important to $\mathrm{AD}$ pathophysiology (i.e., precuneus and posterior cingulate gyrus), there were statistically significant relationships with plasma NF-L and imaging markers of pathology. In addition, there were statistically significant inverse correlations with cognitive performance as measured by the Cambridge Neuropsychological Test Automated Battery (CANTAB) Paired Associate Learning test as well as informant-based measures of memory function, the Observer memory questionnaire-parent form (OMQ-PF). Plasma NF-L is associated with progressive neurodegeneration as well as with declines in cognitive and functional measures in adults with DS and may serve as a useful outcome measure in clinical trials.

The Role of Inflammation and Nerve Growth Factor Dysfunction in Cognitive Decline and AD Pathology in DS

Results from a multisite project between researchers in Canada, USA, and Spain to study the role of nerve growth factor (NGF) metabolism and chronic inflammation in the development of AD in DS were presented. Florencia Iulita (Canada) showed that brains and plasma samples from individuals with DS have higher levels of proNGF (NGF precursor) and MMP-9 activity (NGF degrading enzyme). While an increase in neuroserpin (a tPA inhibitor which prevents proNGF maturation) was observed in frontal cortex, no changes were apparent in plasma. In contrast, levels of the metallo-proteases MMP-1and MMP-3 (which activates MMP-9) as well as of the inflammatory markers TNF- $\alpha$, IL-6, and IL-10 were elevated in plasma from adults with DS, both with and without dementia. The longitudinal within-subjects yearly increase in plasma proNGF correlated with greater cognitive decline at year 2 of follow-up. In addition, higher $A \beta 42$ at

Third International Conference of the T21 Research Society baseline, increased TNF- $\alpha$ at follow-up, lower baseline IL-8, and decrease in IL-8 over follow-up were the strongest predictors of prospective cognitive deterioration. These data support the combination of biomarker analysis with cognitive assessments for the identification of individuals at risk of dementia.

CSF biomarkers effectively contribute to AD diagnoses in the general population. Blood biomarkers with the Single Molecule Array (SIMOA) technology provide a novel opportunity to explore AD pathophysiology using plasma biomarkers. Juan Fortea (Spain) reviewed the evidence on the diagnostic performance of plasma and CSF biomarkers in this population and presented new data on the natural history of fluid AD biomarkers in DS from the Down Alzheimer Barcelona Neuroimaging Initiative (DABNI) cohort, showing the trajectory of all fluid biomarkers in relation with age. The results support the use of these biomarkers in clinical practice and clinical trials [Fortea et al., 2020].

Maria Lioudyno (USA) presented analysis of the progression of AD-like pathology in T21 (T21) neuronal cultures derived from postmortem human fetal cortical samples to define temporal and mechanistic relationships between different pathological processes. Progressive dystrophy of neurites in T21 neurons was accompanied by increased phosphorylated Tau and $A \beta$ (Ser8-pAb) as well as by oxidative stress. As none of these pathological features were prevented by treatment with a gammasecretase inhibitor, $\mathrm{AB}$-independent mechanisms are likely to play equally important pathogenic roles. The results indicate that combination therapies simultaneously targeting amyloidosis, neuroinflammation, oxidative stress and neurotrophic deficiency could successfully prevent neurodegeneration in DS.

\section{$A D$ in the Context of the Trisomy of Hsa21 -}

\section{Preclinical Studies}

William Mobley (USA) pointed to the exciting recent findings of research from preclinical models exploring the how other genes on chromosome 21 may modulate $\mathrm{AD}$ development in DS.

Fei Liu (USA) focused on the role of Dyrk1a on Tau pathology. In the Ts65Dn mouse brain, in which Dyrk1ais overexpressed, phosphorylation of Tau and the ratio of 3R/4R-Tau were increased compared to controls. Moreover, in the brains of people with DS, the DYRK1A level correlated with hyperphosphorylation of tau and the level of 3R-tau. Thus, upregulation of Dyrk1A due to T21 may contribute to tau pathogenesis by phosphorylation of tau and through increased 3R-tau expression. 
Carmen Martinez-Cue (Spain) discussed the role of Dyrkla gene dosage on neurodegeneration in the Ts65Dn mouse model. Normalizing gene dosage of Dyrk1A (from 3 to 2 copies) rescued the density of senescent cells, prevented cholinergic neuron degeneration, reduced APP expression, and reduced $A \beta$ and Tau levels [García-Cerro et al., 2017]. Dr. Martinez-Cue also reported that chronic administration of anti-IL17A partially improved cognitive function, reduced the expression of several pro-inflammatory cytokines and the density of activated microglia, and normalized APP and A $\beta 1-42$ levels in the Ts65Dn mouse model [Rueda et al., 2018]. These data point to the possibility that modulation of neuroinflammation may be a useful therapy for the treatment of $\mathrm{AD}$ in DS.

Using a panel of mouse models of DS, Frances Wiseman (UK) is exploring which Hsa21 genes are sufficient to modulate APP/A $\beta$. She crossed mouse models of DS (Tc1, Dp2Tyb, Dp3Tyb, Dp10Yey and Dp17Yey) with a conventional mouse model of amyloid deposition (J20 $A P P$ transgenic) to determine how an additional copy of the Hsa21 genes affect APP/A $\beta$ biology. T21 (in the absence of increased $A P P$ gene dose) modifies $A \beta$ deposition; the underlying mechanism likely includes modulating the catabolism and resultant aggregation of $A \beta$ via an effect on $A \beta 40 / 42$ ratio [Wiseman et al., 2015, 2018]. These data suggest that people who have DS may have an increased risk of developing $\mathrm{AD}$ not only due to trisomy of $A P P$, but also because trisomy of other chromosome 21 genes.

Tomer Illouz (Israel) reported on a maternal antiA $\beta 1-11$ vaccination strategy for amyloid prevention in the $5 x F A D$ model of amyloid deposition. Transgenic pups that had maternally induced anti-A $\beta$ antibodies, were actively vaccinated with either the $A \beta 1-11$ or sham vaccine. Maternal vaccination provided passive immunization, as anti- $\mathrm{A} \beta$ antibodies were present in the circulation prenatally at E18 and postnatally at P28. Maternal vaccination alone, and maternal vaccination added to active postnatal vaccination, each reduced cortical $A \beta$ levels to similar extents, suggesting that early maternal immunization is sufficient to elicit $A \beta$ clearance. Maternal anti$A \beta$ immunization confers long-term beneficial effects in mice, likely by mediating A $\beta$-clearance. The exciting possibility was suggested that prenatal vaccination may be considered as means to prevent AD in DS.

Dean Nizetic (Singapore and UK) discussed the role of the Hsa21 gene BACE2 in the suppression of AD neuropathology in iPSC-derived cerebral organoid DS model. BACE2 is an aspartic-like protease, which can cleave APP as a $\beta$ - and $\theta$-secretase, and $A \beta$. T21 organoids secreted increased BACE2 product peptides compared to isogenic euploid and duplication of the APP locus controls. Using CRISPR/Cas9 gene editing, Dr. Nizetic's team corrected the BACE2 gene copy number from 3 to 2 in T21 organoids, and this decreased the relative abundance of BACE2related $A \beta$ cleavage fragments and surprisingly triggered early and accelerated AD-like pathology in T21 organoids [Alić et al., 2020] including insoluble amyloid deposits, and pathologically altered Tau. Results suggest increased $B A C E 2$ may slow the development of AD neuropathology in DS.

In his plenary talk, André Strydom (UK) summarized findings from the London DS consortium (LonDownS), one of the first large-scale multidisciplinary studies exploring the causative mechanisms and predictive phenotypes associated with AD in DS. The LonDownS team showed that decline in memory abilities of DS individuals may start during their early 40s [Startin et al., 2019], several years before mean diagnosis of dementia at age 54 [Sinai et al., 2018]. Yet people with DS have been largely excluded from clinical trials due to a lack of reliable data on how the disease progresses, and concerns about reliability of outcome measures. Dr. Strydom outlined how new findings on plasma biomarkers in DS, particularly neurofilament light, a marker of neurodegeneration [Strydom et al., 2018b], in combination with sensitive measures of cognitive decline and accounting for the effect of genetic factors such as APOE could help to define the stages of dementia [Firth et al., 2018] and transform the prospects for individuals with DS by enabling clinical trials of treatment to prevent or delay AD [Strydom et al., 2018a]. Neuropathological studies of brain material from DS individuals and those from duplication of only the APP region on Hsa21 suggest some protection against severity of cerebral amyloid angiopathy [Mann et al., 2018].

\section{Endpoints for Clinical Trials}

Clinical geneticist Clotilde Mircher (France) presented results from the ACTHYF single-center, randomized, double-blind, placebo-controlled phase 3 clinical trial assessing the efficacy of L-thyroxin and/or folinic acid treatment on psychomotor development in 143 infants and toddlers with DS, 6-18 months old. Exclusion criteria included congenital heart disease, neurologic abnormalities, problems with vision and a history of premature birth. The trial included 4 treatment arms: (1) placebo, (2) folinic acid, (3) L-thyroxin, or (4) folinic acid + L-thyroxin. The primary efficacy endpoint concerned change in the global development quotient (Griffiths Mental Devel- 
opment Scale) after 1 year of treatment versus baseline. The results show no effect of treatment and no differences between males and females. Despite these negative results, Dr. Mircher highlighted important lessonslearned from the study, including issues of the inclusion rate, psychometric evaluation and variability in outcome measures that should be considered for future clinical trials.

Bessy Benejam (Spain) discussed proposed improvements in the Cambridge Cognitive Examination for Older Adults with DS (CAMCOG-DS) assessment tool to enhance the applicability of this neuropsychological test in people with DS. Dr. Benejam explained why specific items in the original CAMCOG-DS relating to, among others, memory, abstract thinking and language were problematic, and showed the pragmatic solution developed by multiple European neuropsychologists. An improved scoring system for the drawing and copying task (praxis) was proposed as well. Importantly, Dr. Benejam showed data demonstrating that the CAMCOG-DS is applicable in healthy (asymptomatic) people with DS with mild (100\%) and moderate (97\%) intellectual disability, and in $56 \%$ of those with a severe intellectual disability. However, the test is not applicable in $44 \%$ of the severely and virtually none of the profoundly disabled individuals. Other measures are needed in this subpopulation. With the onset of AD dementia, the applicability of the CAMCOG-DS decreased in all groups (level of intellectual disability), but remained very useful.

James Hendrix (USA) introduced the DS Clinical Trials Network, a network of 11 US sites with greater than 5,000 individuals with DS aims to (1) advance effective treatments, (2) translate basis research into more DS clinical trials, and (3) significantly and tangibly improve care for people with DS. In June 2019, the first study was launched within this network, the Longitudinal Investigation for Enhancing DS Research (Life-DSR) study. This observational natural history study, in which 270 adults with DS ( $\geq 25$ years) will be followed over the course of 2 years, aims to characterize an adult population and develop sensitive/validated assessment instruments for future prevention clinical trials for AD. Plasma samples will be collected and stored for future biomarker research. Cognitive and functional tests will be performed at each visit (baseline, 1- and 2-year follow-up).

Laura Videla (Spain) showed that the CAMCOG-DS battery and Cued Recall Test (CRT) detected cognitive decline related to AD with good sensitivity and specificity in people with DS with a mild or moderate intellectual disability, but not in those with severe or profound intellectual disability (limited applicability). Videla sub-

Third International Conference of the T21 Research Society sequently define optimal cut-off points for these tests using 2 approaches (normative data and ROC).

Meifang Xiao, (USA) presented results showing reduced levels of Neuronal pentraxin 2 (NPTX2) that plays a role in parvalbumin-interneuron-mediated inhibition, in brain and CSF of both $\mathrm{AD}$ and DS individuals. Interestingly, CSF NPTX2 also correlated with specific cognitive performance measures and predicted cognitive decline in AD individuals. In DS, CSF NPTX2 was found to correlate with glucose metabolism (FDG-PET) and cortical thickness (MRI).

\section{Fostering the New Generation of Researchers}

Nearly $40 \%$ of attendees were students and post-doctoral fellows that represent the next generation of DS researchers. To fulfill the mission of T21RS to stimulate young members to carry out high-level research and recognize their merit with financial support, the Annette Karmiloff-Smith and Michael Harpold Thesis Awards were presented to honor the memory of influential T21RS members. Dr. Renata Bartesaghi (Italy) presented the Annette Karmiloff-Smith Dissertation Award to Nadine Aziz (USA), whose $\mathrm{PhD}$ thesis was entitled "Histological, cellular, and molecular abnormalities in forebrain and spinal cord of three distinct mouse models of DS" [Aziz et al., 2018]. Eric Hamlett (USA) received the Michael Harpold Dissertation Award for his PhD thesis entitled "Investigations at the crossroads of DS and AD" [Hamlett et al., 2017, 2019, 2020].

\section{Science and Society Program}

The T21RS Committee for Science \& Society session was devoted to exchanging perspectives between scientists and family members. Scientific attendees of the conference were joined by nearly a hundred family members and people with DS. Peter De Deyn (The Netherlands) opened the symposium by stressing the importance of deepening collaboration between scientists and family associations.

Hampus Hillerstrom (USA) presented results from a family survey about DS research completed by 256 parents in 8 countries. Consistent with other surveys, the most prominent medical concerns raised by parents related to $\mathrm{AD}$ were cognition, sleep, speech, and behavior. However, the survey also showed that a substantial number of parents have concerns about risks, transparency 
and intent of studies. Reasons (not) to participate in studies relate to e.g., distance to the research study site, access to results, the study purpose and the invasiveness of procedures. Finally, Hillerstrom asked the audience to think along about the issue of including minorities in research, such as families with lower income or from non-European descent, which remains a challenge and apparently requires another recruitment strategy.

Juan Fortea (Spain) focused on AD in adults with DS. He stated that we are more aware of comorbidities in children with DS than those later in life. Fortea presented the innovative health plan for adults with DS in Catalonia, which combines annual screenings with the possibility to participate in research. Biomarker studies are a major focus of this cohort study because (1) the diagnostic performance of biomarkers might be different from the general population, (2) it contributes to understanding $\mathrm{AD}$ pathophysiology in DS, and (3) it may allow for patient selection and monitoring of treatment efficacy in clinical trials. Fortea briefly highlighted the promising diagnostic performance of the classic $\mathrm{AD}$ biomarkers (Aß1-42, $t-$ tau, p-tau) in cerebrospinal fluid as well as the finding that the neurofilament light (NfL) concentration in plasma may be useful as well [Fortea et al., 2020]. Since blood sampling is less invasive than a lumbar puncture, these plasma biomarkers may change future clinical practice.

Michelle Whitten (USA) gave an overview of how the Global DS Foundation contributes to scientific research, medical care, education, and advocacy. One of the main achievements is the establishment of the Linda Crnic Institute for DS, established in 2008 in Denver that hosts over 200 scientists working on AD, cognition, immunology, stem cells, genetics, and leukemia. The foundation also promotes research by lobbying for DS research funding in Washington D.C. and was instrumental in increasing DS research funding at the National Institutes of Health (USA).

An interactive debate led by Peter De Deyn (The Netherlands) focused on (1) donation of blood and tissue among including postmortem brain donation - for scientific research, (2) communication of study results to participants, and (3) whether studies should have direct benefit to participants. The panel consisted of Diana Bianchi (USA), Lotta Granholm (USA), Elizabeth Head (USA), Alain Dekker (The Netherlands), Juan Fortea (Spain), and Sebastian Videla (Spain). The majority of attendees were in favor of participating in research (even without direct benefit) as long as the aim is clear, the procedure is well adapted and the approach is respectful. For example, one individual with DS stated: "I think about my death. I want to donate my brain." A mother responded by saying, "It is easier to say, than to do. It's more about emotion [when the moment is there]." Acknowledging that such decisions would best be made in advance by the person with DS and his/her family (advanced directive), it was noted that the audience had a pro-research attitude. The panel stressed that collection of blood and tissue samples is of utmost importance. Without samples, researchers will not be able to investigate mechanisms and find novel targets for e.g., cognitive deficits and $\mathrm{AD}$ in people with DS.

Jesús Florez (Spain) addressed the topic of autonomy of people with DS and described various circumstances that play a role and what we should (and should not) expect from autonomy. Florez stressed that autonomy cannot be achieved by an easy, single recipe, but that promoting autonomy requires finding a balance, taking into account someone's strengths and weaknesses, actual capabilities and physical and emotional vulnerability. It is not only about promoting decision making, but also about teaching people with DS to accept consequences of their decisions. It is a matter of rights and responsibilities. For each unique individual, the recipe may be different.

The symposium ended with the presentation of the Montserrat Trueta Human Rights Assembly from the Catalan DS Foundation (Barcelona, Spain). Three prominent members of this Assembly who have DS, Andy Trias (president), Montserrat Vilarrasa (secretary), and Ana Rodriguez (responsible of the agenda), enthusiastically presented the work of the Assembly and their own role in this societal initiative.

The Third T21RS International Conference promoted scientific exchanges and defined the most promising research at the basic, translational and clinical levels. The rapid pace of progress that has taken place in the last few years demands effective communication between scientists and clinicians working in the field. The vibrant content of the meeting shows that our community continues to thrive.

\section{Acknowledgement}

The conference was supported by the Center for Genomic Regulation (Barcelona, Spain) and enthusiastic help from volunteering students and people with DS. T21RS is the first international nonprofit research society uniting scientists and clinicians studying DS from all over the world. The society was established on the $17^{\text {th }}$ of April 2014, in Groningen, The Netherlands. During the conference, Dr. Mara Dierssen (president), Dr. Marie-Claude Potier (secretary), Dr. Alain D. Dekker (treasurer), and the chairmen of the standing committees presented the efforts and initiatives of 
the society during the annual General Assembly. Conference information, annual reports, and more information about the society and the Conference is available at www.t21rs.org.

\section{Conflict of Interest Statement}

The authors declare no conflicts of interest.

\section{Funding Sources}

The T21RS International Conference 2019 in Barcelona was supported by generous donations from La Caixa Foundation (Barcelona, Spain) that offered the conference venue "Cosmo Caixa" science museum, Fondation Jérôme Lejeune (Paris, France), Lu-
mind-IDSC Foundation (Burlington, MA, USA), Global DS Foundation (Denver, CO, USA), Eunice Kennedy Shriver National Institute of Child Health \& Human Development of the National Institutes of Health under Award Number R13HD098868 (USA), Spanish DS Federation "Down España” (Madrid, Spain), Fundación Iberoamericana Down21 (Santander, Spain), National DS Society (New York, NY, USA), Fondation AMIPI-Bernard Vendre (Cholet, France), The Co. of Biologists (Cambridge, UK), and The Prous Institute for Biomedical Research (Barcelona, Spain), Catalan DS Foundation (Barcelona, Spain).

\section{Author Contributions}

All of the authors served as Chairs of symposia at the meeting and wrote relevant sections of the manuscript.

\section{References}

Alić I, Goh PA, Murray A, Portelius E, Gkanatsiou E, Gough G, et al. Patient-specific Alzheimerlike pathology in trisomy 21 cerebral organoids reveals $\mathrm{BACE} 2$ as a gene dose-sensitive $\mathrm{AD}$ suppressor in human brain. Mol Psychiatry. 2020.

Antonaros F, Ghini V, Pulina F, Ramacieri G, Cicchini E, Mannini E, et al. Plasma metabolome and cognitive skills in Down syndrome. Sci Rep. 2020;10(1):10491.

Aziz NM, Guedj F, Pennings JLA, Olmos-Serrano JL, Siegel A, Haydar TF, et al. Lifespan analysis of brain development, gene expression and behavioral phenotypes in the Ts1Cje, Ts65Dn and $\mathrm{Dp}(16) 1 /$ Yey mouse models of Down syndrome. Dis Model Mech. 2018;11(6): dmm031013.

Coppus A. Comparing Generational Differences in Persons With Down Syndrome. J Policy Pract Intel. 2017;14(2):118-23.

Cornacchia M, Sethness J, Alapat P, Lin YH, Peacock C. The Prevalence of OSA Among an Adult Population With Down Syndrome Referred to a Medical Clinic. Am J Intellect Dev Disabil. 2019;124(1):4-10.

Czerminski JT, Lawrence JB. Silencing Trisomy 21 with XIST in Neural Stem Cells Promotes Neuronal Differentiation. Dev Cell. 2020;52: 294-308 e3.

Delabar JM, Allinquant B, Bianchi D, Blumenthal T, Dekker A, Edgin J, et al. Changing Paradigms in Down Syndrome: The First International Conference of the Trisomy 21 Research Society. Mol Syndromol. 2016;7(5):251-61.

Dierssen M, Fructuoso M, Martínez de Lagrán M, Perluigi M, Barone E. Down Syndrome Is a Metabolic Disease: Altered Insulin Signaling Mediates Peripheral and Brain Dysfunctions. Front Neurosci. 2020;14:670.

Dodd K, Watchman K, Janicki MP, Coppus A, Gaertner C, Fortea J, et al. Consensus statement of the international summit on intellectual disability and Dementia related to postdiagnostic support. Aging Ment Health. 2018; 22(11):1406-15.
Esbensen AJ, Hooper SR, Fidler D, Hartley SL, Edgin J, d'Ardhuy XL, et al. Outcome Measures Working G: Outcome Measures for Clinical Trials in Down Syndrome. Am J Intellect Dev Disabil. 2017;122:247-81.

Finkbeiner S. The Autophagy Lysosomal Pathway and Neurodegeneration. Cold Spring Harb Perspect Biol. 2020;12(3):a033993.

Firth NC, Startin CM, Hithersay R, Hamburg S, Wijeratne PA, Mok KY, et al. Aging related cognitive changes associated with Alzheimer's disease in Down syndrome. Ann Clin Transl Neurol. 2018;5(6):741-51.

Fisher EMC, Bannerman DM. Mouse models of neurodegeneration: Know your question, know your mouse. Sci Transl Med. 2019; 11(493):eaaq1818.

Fortea J, Vilaplana E, Carmona-Iragui M, Benejam B, Videla L, Barroeta I, et al. Clinical and biomarker changes of Alzheimer's disease in adults with Down syndrome: a cross-sectional study. Lancet. 2020;395(10242): 1988-97.

Galati DF, Sullivan KD, Pham AT, Espinosa JM, Pearson CG. Trisomy 21 Represses Cilia Formation and Function. Dev Cell. 2018;46(5): 641-650.e6.

García-Cerro S, Rueda N, Vidal V, Lantigua S, Martínez-Cué C. Normalizing the gene dosage of Dyrk1A in a mouse model of Down syndrome rescues several Alzheimer's disease phenotypes. Neurobiol Dis. 2017;106: 76-88.

Giménez S, Videla L, Romero S, Benejam B, Clos S, Fernández S, et al. Prevalence of Sleep Disorders in Adults With Down Syndrome: A Comparative Study of Self-Reported, Actigraphic, and Polysomnographic Findings. J Clin Sleep Med. 2018;14(10):1725-33.

Hamlett ED, Goetzl EJ, Ledreux A, Vasilevko V, Boger HA, LaRosa A, et al. Neuronal exosomes reveal Alzheimer's disease biomarkers in Down syndrome. Alzheimers Dement. 2017;13(5):541-9.
Hamlett ED, LaRosa A, Mufson EJ, Fortea J, Ledreux A, Granholm AC. Exosome release and cargo in Down syndrome. Dev Neurobiol. 2019;79(7):639-55.

Hamlett ED, Ledreux A, Gilmore A, Vazey EM, Aston-Jones G, Boger HA, et al. Inhibitory designer receptors aggravate memory loss in a mouse model of down syndrome. Neurobiol Dis. 2020;134:104616.

Hwang S, Williams JF, Kneissig M, Lioudyno M, Rivera I, Helguera P, et al. Suppressing Aneuploidy-Associated Phenotypes Improves the Fitness of Trisomy 21 Cells. Cell Rep. 2019; 29(8):2473-2488.e5.

Iaccarino HF, Singer AC, Martorell AJ, Rudenko A, Gao F, Gillingham TZ, et al. Gamma frequency entrainment attenuates amyloid load and modifies microglia. Nature. 2016; 540(7632):230-5.

Jiang J, Jing Y, Cost GJ, Chiang JC, Kolpa HJ, Cotton AM, et al. Translating dosage compensation to trisomy 21. Nature. 2013;500(7462): 296-300.

Kalebic N, Gilardi C, Stepien B, Wilsch-Bräuninger M, Long KR, Namba T, et al. Neocortical Expansion Due to Increased Proliferation of Basal Progenitors Is Linked to Changes in Their Morphology. Cell stem cell. 2019;24(4): 535-550e9.

Kazuki Y, Gao FJ, Li Y, Moyer AJ, Devenney B, Hiramatsu K, et al. A non-mosaic transchromosomic mouse model of down syndrome carrying the long arm of human chromosome 21. ELife. 2020;9:e56223.

Krinsky-McHale SJ, Silverman W. Dementia and mild cognitive impairment in adults with intellectual disability: issues of diagnosis. Dev Disabil Res Rev. 2013;18(1):31-42.

Krinsky-McHale SJ, Jenkins EC, Lee JH, Schupf N, Zigman WB, Silverman W. Individual differences in lifelong abilities must be considered for staging of early AD. England: Alzheimer's Association International ConferenceLondon; 2017. 
Laurent AP, Siret A, Ignacimouttou C, Panchal K, Diop MB, Jenni S, et al. Constitutive Activation of RAS/MAPK Pathway Cooperates with Trisomy 21 and Is Therapeutically Exploitable in Down Syndrome B-cell Leukemia. Clin Cancer Res. 2020;26(13):3307-18.

Mann DMA, Davidson YS, Robinson AC, Allen N, Hashimoto T, Richardson A, et al. Patterns and severity of vascular amyloid in Alzheimer's disease associated with duplications and missense mutations in APP gene, Down syndrome and sporadic Alzheimer's disease. Acta Neuropathol. 2018;136(4):569-87.

Martorell AJ, Paulson AL, Suk HJ, Abdurrob F, Drummond GT, Guan W, et al. Multi-sensory Gamma Stimulation Ameliorates Alzheimer's-Associated Pathology and Improves Cognition. Cell. 2019;177(2):256-271e22.

McCarron M, McCallion P, Coppus A, Fortea J, Stemp S, Janicki $M$, et al. Supporting advanced dementia in people with Down syndrome and other intellectual disability: consensus statement of the International Summit on Intellectual Disability and Dementia. J Intellect Disabil Res. 2018;62(7):617-24.

Miranda AM, Herman M, Cheng R, Nahmani E, Barrett G, Micevska E, et al. Excess Synaptojanin 1 Contributes to Place Cell Dysfunction and Memory Deficits in the Aging Hippocampus in Three Types of Alzheimer's Disease. Cell Rep. 2018a;23(10):2967-75.

Miranda AM, Lasiecka ZM, Xu Y, Neufeld J, Shahriar S, Simoes S, et al. Neuronal lysosomal dysfunction releases exosomes harboring APP C-terminal fragments and unique lipid signatures. Nat Commun. 2018b;9(1):291.

Nawa N, Hirata K, Kawatani K, Nambara T, Omori S, Banno K, et al. Elimination of protein aggregates prevents premature senescence in human trisomy 21 fibroblasts. PloS One. 2019;14(7):e0219592.
Ovchinnikov DA, Korn O, Virshup I, Wells CA, Wolvetang EJ. The Impact of APP on $\mathrm{Al}$ zheimer-like Pathogenesis and Gene Expression in Down Syndrome iPSC-Derived Neurons. Stem Cell Reports. 2018;11(1):32-42.

Parra V, Altamirano F, Hernández-Fuentes CP, Tong D, Kyrychenko V, Rotter D, et al. Down Syndrome Critical Region 1 Gene, Rcan1, Helps Maintain a More Fused Mitochondrial Network. Circ Res. 2018;122(6):e20-33.

Ponroy Bally B, Farmer WT, Jones EV, Jessa S, Kacerovsky JB, Mayran A, et al. Human iPSCderived Down syndrome astrocytes display genome-wide perturbations in gene expression, an altered adhesion profile, and increased cellular dynamics. Hum Mol Genet. 2020;29(5):785-802.

Powers RK, Culp-Hill R, Ludwig MP, Smith KP, Waugh KA, Minter R, et al. Trisomy 21 activates the kynurenine pathway via increased dosage of interferon receptors. Nat Commun. 2019;10(1):4766.

Real R, Peter M, Trabalza A, Khan S, Smith MA, Dopp J, et al. In vivo modeling of human neuron dynamics and Down syndrome. Science. 2018;362(6416):eaau1810.

Reeves RH, Delabar J, Potier MC, Bhattacharyya A, Head E, Lemere C, et al. Paving the Way for Therapy: The Second International Conference of the Trisomy 21 Research Society. Mol Syndromol. 2019;9(6):279-86.

Rueda N, Vidal V, García-Cerro S, Narcís JO, Llorens-Martín M, Corrales A, et al. Anti-IL17 treatment ameliorates Down syndrome phenotypes in mice. Brain Behav Immun. 2018; 73:235-51.

Shaw PR, Klein JA, Aziz NM, Haydar TF. Longitudinal neuroanatomical and behavioral analyses show phenotypic drift and variability in the Ts65Dn mouse model of Down syndrome. Dis Model Mech. 2020;13(9): dmm046243.
Sinai A, Mokrysz C, Bernal J, Bohnen I, Bonell S, Courtenay K, et al. Predictors of Age of Diagnosis and Survival of Alzheimer's Disease in Down Syndrome. J Alzheimers Dis. 2018; 61(2):717-28.

Stamoulis G, Garieri M, Makrythanasis P, Letourneau A, Guipponi M, Panousis N, et al. Single cell transcriptome in aneuploidies reveals mechanisms of gene dosage imbalance. Nat Commun. 2019;10(1):4495.

Startin CM, Hamburg S, Hithersay R, Al-Janabi T, Mok KY, Hardy J, et al. Cognitive markers of preclinical and prodromal Alzheimer's disease in Down syndrome. Alzheimers Dement. 2019;15(2):245-57.

Strydom A, Coppus A, Blesa R, Danek A, Fortea J, Hardy J, et al. Alzheimer's disease in Down syndrome: An overlooked population for prevention trials. Alzheimers Dement (NY). 2018a;4:703-13.

Strydom A, Heslegrave A, Startin CM, Mok KY, Hardy J, Groet J, et al. The LonDown SC: Neurofilament light as a blood biomarker for neurodegeneration in Down syndrome. Alzheimers Res Ther. 2018b;10(1):39.

Tramutola A, Lanzillotta C, Di Domenico F, Head E, Butterfield DA, Perluigi M, et al. Brain insulin resistance triggers early onset Alzheimer disease in Down syndrome. Neurobiol Dis. 2020;137:104772.

Wiseman FK, Al-Janabi T, Hardy J, KarmiloffSmith A, Nizetic D, Tybulewicz VL, et al. A genetic cause of Alzheimer disease: mechanistic insights from Down syndrome. Nat Rev Neurosci. 2015;16(9):564.

Wiseman FK, Pulford LJ, Barkus C, Liao F, Portelius E, Webb R, et al. Trisomy of human chromosome 21 enhances amyloid- $\beta$ deposition independently of an extra copy of APP. Brain. 2018;141(8):2457-74. 